\title{
La labor de los editores en la enseñanza de lenguas extranjeras en el siglo XIX
}

\section{M. a Ángeles García Aranda}

\section{OpenEdition}

\section{Journals}

Edición electrónica

URL: https://journals.openedition.org/cher/3297

DOI: $10.4000 /$ cher.3297

ISSN: 2803-5992

\section{Editor}

Presses universitaires de Strasbourg

\section{Edición impresa}

Fecha de publicación: 27 junio 2017

Paginación: 139-152

ISBN: 978-2-86820-961-0

ISSN: 1968-035X

\section{Referencia electrónica}

M. ${ }^{a}$ Ángeles García Aranda, «La labor de los editores en la enseñanza de lenguas extranjeras en el siglo xIx», reCHERches [En línea], 18 | 2017, Publicado el 01 diciembre 2021, consultado el 18 diciembre 2021. URL: http://journals.openedition.org/cher/3297 ; DOI: https://doi.org/10.4000/cher.3297

\section{cc) () (2)}

Ce(tte) œuvre est mise à disposition selon les termes de la Licence Creative Commons Attribution Pas d'Utilisation Commerciale - Partage dans les Mêmes Conditions 4.0 International. 


\title{
La labor de los editores en la enseñanza de lenguas extranjeras en el siglo $X^{\prime} X^{\prime}$
}

\author{
M. a Ángeles García ArandA*
}

\section{Introducción: la casa Truchy-Leroy}

Las circunstancias políticas, económicas y sociales en que se desarrolló el siglo XIX motivaron el interés por el aprendizaje de lenguas extranjeras -dentro y fuera de las aulas- y, como consecuencia de ello, el aumento de la edición y publicación de manuales de enseñanza. Surgen, en ese contexto, los métodos Ollendorf, Ahn, Berlitz, Dufief, Gouin, Robertson, Hugo o Hossfeld, y también, dados los beneficios económicos que proporcionaba la industria del libro, otros textos destinados a la enseñanza de segundas lenguas creados por editores o impresores (Choppin 1992, Cooper-Richet 1999 y 2002, Sánchez Pérez 1992).

En Francia ${ }^{2}$, en donde «l'introduction des langues vivantes dans les programmes de l'enseignement secondaire, à titre facultatif en 1829, puis comme discipline obligatoire à partir de 1838» (Choppin 1995: 19), la labor de editores e impresores fue determinante en la publicación de manuales para la enseñanza de segundas lenguas, pues estuvieron detrás de muchos de los textos didácticos que se reeditaron y reimprimieron a lo largo de la centuria para que los franceses pudieran aprender otras lenguas modernas. Estos manuales han quedado al margen de la historia de la enseñanza de lenguas y de la historiografía lingüística, que se han ocupado de los diferentes métodos de enseñanza (método tradicional,

* Profesora titular del área de Lengua española en el Departamento de Lengua española y Teoría de la literatura y Literatura comparada de la Universidad Complutense de Madrid.

1 Este trabajo se enmarca dentro del Proyecto de Investigación «Biblioteca Virtual de la Filología Española. Fase II. Consolidación, mejora y ampliación de los datos y de la web. Estudio de los materiales contenidos» (FFI2014-5381-P).

2 Algunos de los trabajos que se han ocupado de los materiales de enseñanza de francés en España y de español en Francia en el siglo xıx son: Bruña Cuevas (2008), Cazorla (2002), Fernández Fraile/Suso López (1999), Quemada (1967). 
método gramática-traducción, método natural...), su historia, su evolución, sus novedades y sus principales representantes (Ahn, Ollendorf, Otto-Sauer, Ochoa, Sotos Ochando, Novísimo Chantreau). Junto a ellos hubo otros menos conocidos, menos famosos, pero que, dada la cantidad de ediciones, dada la diversidad de materiales utilizados y dado el número de lenguas a los que fueron traducidos, merecen ser considerados y valorados. Uno de ellos fue J.H. Truchy ${ }^{3}$, dueño de la imprenta parisina "francesa e inglesa» sita en el Boulevard des Italianes -y sus sucesores, los hermanos Leroy-, quien "penetrado de la utilidad de las lenguas extrangeras, hace treinta años que me estoy consagrando a su propagación y el catálogo de mi casa puede atestiguar los esfuerzos que he hecho para alcanzar este resultado» (Lopes 1864: 7). A lo largo de varias décadas, Truchy creó un método de enseñanza de lenguas que adaptó - gracias a la colaboración de varios profesores de lenguas ${ }^{4}-$ primero al inglés y al español y, con el paso del tiempo, también al italiano, al portugués, al alemán y al ruso ${ }^{5}$.

Pero el método Truchy, pese a las bondades que pregona el editor ${ }^{6} \mathrm{y}$ pese a que fue sancionado por la Universidad de Francia y por el Consejo Real de

3 En trabajos anteriores me he ocupado de algunas de las obras de la editorial Truchy (2012a, 2012b y 2014); en esta ocasión presento el método de Truchy de enseñanza de lenguas de forma íntegra, lo que permitirá valorar adecuadamente la labor y la aportación del editor a la historia de la enseñanza de lenguas.

4 Truchy, por ejemplo, contó con José M.a Moralejo, «doctor en Teología por la Universidad de Alcalá y maestro de sordomudos», con José M. Lopes, «bachiller en ciencias y en letras, profesor de la Asociación filotécnica y de la propagación de la instrucción elemental» y con Pablo Valdemoros y Álvarez, "profesor de español en la escuela municipal, en la Escuela especial de comercio y en la Escuela de Artes industriales», para adaptar textos en español; con Percy Sadler, «miembro de la Sociedad gramatical de Paris» para el inglés o con Luigi Sforzosi para el italiano. Por esta razón, Alain Choppin (1992: 58) insiste en diferenciar en este siglo la labor de autores, impresores y editores, «les professionnels du livre peuvent désormais choisir leurs auteurs, fixer leurs émoluments, imposer à leurs textes les modifications qu'ils jugent opportunes pour l'obtention de l'approbation universitaire».

5 En este sentido concluye Alain Choppin (1995: 19), tras analizar la historia y evolución de los manuales escolares de español en Francia de 1789 a nuestros días, que, una vez integradas las lenguas modernas en los planes de estudio, « 43 manuels sont édités en moyenne par décennie de 1840 à 1859 contre 32 de 1820 à 1839 . La priorité accordée à l'enseignement des langues vivantes au lendemain de la guerre de 1870 profitant essentiellement à l'allemand -et, dans une moindre mesure, à l'anglais- n'a aucune incidence sur la production des manuels d'espagnol».

6 Truchy defiende que el perfecto método de enseñanza de lenguas debe ser «une pratique éclairée par la théorie ou des règles appuyés par de nombreux exemples et des exercices», esto es, que no incurra en los defectos de los manuales habituales: «L'étude des langues étrangères a toujours été faite au point de vue de deux systèmes, qui, bien que distincts en apparence, devraient pourtant être inseparables l'un de l'autre; et cependant ils partagent les professeurs en deux camps. Les uns, partisans trop zélés du système classique, se basent presque exclusivament sur la grammaire et forment purement des littérateurs instruits dans une langue étrangère, voire même des écrivains, mais qui, pour parler cette langue, sont obliés de recommencer une série d'exercices pratiques. Les autres, apprenant simplement des phrases et des mots, 
Instrucción Pública quien los recomendó para las escuelas públicas, no es ni nuevo ni original en la historia de la enseñanza de lenguas: las gramáticas, los vocabularios o las guías de conversación que salen de sus prensas exponen contenidos tradicionales pero adaptados a la nueva realidad y a los nuevos usuarios. Las siguientes páginas se detienen en algunos de los textos que configuran este método, su contenido, sus fuentes y sus objetivos, con el fin de mostrar cómo editores e impresores contribuyeron a la enseñanza de lenguas en la Francia decimonónica.

\section{La gramática}

Las gramáticas elaboradas en la casa Truchy son sencillas y breves, con numerosos ejercicios que facilitan el aprendizaje de la lengua a sus destinatarios; en el prefacio de ellas se lee: «elle est simple, claire, facile et à la portée de tous. Il rend compte de tout, et ne laisse aucune difficulté sans la résoudre; au besoin, il multiplie les exemples pour ne rien laisser dans le vague: aussi l'élève est-il étonné lui-même de la rapidité de ses progrès» (Lopes 1866: VI).

Con estas premisas, Truchy y los hermanos Leroy publican, para casi todos los niveles educativos, diferentes textos gramaticales:

- la Nouvelle méthode pratique de la langue anglaise, renfermant des Exercices simplifiés sur les règles de la grammaire, suivis d'un Dictionnaire anglais-français de tous les mots employés dans l'ouvrage (firmada por Stebbing), Le Maître d'anglais (por Alfred-G. Havet), English Verbs and English Prepositions. Rational of the People's English or Specimens of the Free and Easy. L'anglais raisonné jusqu'en ses libres allures (por Ch. Fleming), los Études progressives de langue anglaise, divisées en leçons pratiques suivies d'un Recueil de dialogues, formant un Cours d'études de première année (S. Johnson), la Méthode pratique et interlinéaire de langue anglaise (por A. Brown) y Le Vade-mecum anglais, méthode facile et infaillible pour apprendre promptement la langue anglaise (por FournelDesfontaines) para el inglés;

- la Guide pratique de la langue italienne (por Luigi Sforzosi), la Grammaire italienne, élémentaire, analytique et raisonnée, suivie d’un aperçu de la versification italienne (por G. Robello) y la Nouvelle méthode pratique de la langue italienne (por Cardelli) para enseñar italiano;

- la Nouvelle méthode pratique de la langue portugaise (por Soares da Silva) para el portugués;

- los Rudiments de la langue allemande à l'usage des classes élémentaires (por A.Stahl), la Nouvelle méthode pratique de la langue allemande

mêlés çà et là de quelques règles imparfaites, parviennet à faire parler l'élève assez promptement, mais presque toujours d'une manière incorrecte; de là la nécessité qu'ils éprouvent d'entreprendre sérieusemment l'étude des régles gramaticales, de la syntaxe surtout, qui est la logique d'une langue» (Lopes 1873: II). 
(firmada por Werner Kamps), el Tableau du genre de tous les substantifs de la langue allemande (por E. Winter), Les verbes irréguliers de la langue allemande (por K. de Feld) o el Syllabaire allemand et français (por L. Meyer $)^{7}$ para quienes desearan aprender alemán.

Para el español, Truchy publicó la Nouvelle grammaire pratique et raisonnée de la langue espagnole (1866) y la Nouvelle méthode pratique de la langue espagnole (1873), firmadas ambas por José M.Lopes. La primera es una gramática de español para extranjeros, redactada en francés y compuesta por explicaciones gramaticales contrastivas francés-español seguidas de listas de vocabulario y frases en ambas lenguas para ejercitar la traducción directa, version, y la traducción inversa, thème ${ }^{8}$, es decir, un texto poco original en el que la concepción de la gramática (formada por prosodia, partes de la oración, sintaxis y ortografía), las definiciones de las categorías gramaticales o los ejercicios que los acompañan habían aparecido con anterioridad. Y es que, pese a las novedades que acarreó en materia pedagógica la nueva centuria, seguían vigentes en muchos casos los modelos didácticos heredados de la antigüedad clásica y el aprendizaje de una lengua continuaba descansando en «el estudio de las reglas, la traducción, la conversación y la lectura de buenas obras, sistema y principios ya sobradamente conocidos y puestos en práctica por la mayoría de manualistas del presente siglo» (Sánchez Pérez 1992: 177). Con la ayuda de otros "grammairiens modernes» y de «l'Académie espagnole», confecciona una gramática que estructura en dos partes, a saber, pronunciación y partes del discurso 9 , por un lado, y sintaxis (dado que es necesario «examiner leur emploi [el de las clases de palabras], la place qu'elles doivent tenir, le rôle, en fin, qu'elles jouent dans une phrase», Lopes 1866: 387) y ortografía, por otro.

La Nouvelle grammaire no es, por tanto, muy diferente a otras gramáticas coetáneas, si bien es necesario reconocer que los manuales de la casa Truchy eran claros, sencillos y concisos, pues, además de simplificar al máximo la teoría lingüística, incluían abundantes ejercicios, resúmenes, listas de palabras (sobre todo, en el capítulo dedicado al verbo), versions o traducciones directas y thèmes o traducciones inversas, cuadros aclaratorios, y numerosas alusiones al uso real de la lengua. Ese es el gran mérito de este manual, como también reconoce Bescherelle en las últimas páginas del prefacio:

7 Esta información procede del catálogo disponible en el Institut français de l'éducation, http://www.inrp.fr/emma/web/index.php

8 La inclusión de prácticas y traducciones sobre los temas gramaticales tratados se disponen tras cada una de las lecciones, y no al final del volumen como en otras obras.

9 Distingue diez partes del discurso o clases de palabras: artículo, nombre o sustantivo, adjetivo, pronombre, verbo, participio, adverbio, preposición, conjunción e interjección; que pueden dividirse, a su vez, en variables (las seis primeras) e invariables (las cuatro últimas), sistema que curiosamente «es posterior al de nueve clases de palabras» y que es el defendido por la edición de la Gramática de la Real Academia Española de 1870 (Calero Vaquera 1986). 
Puis, viennent les parties $d u$ discours, qui sont toutes traitées d'une manière fort remarquable. L'auteur ne donne que les règles principales, afin de ne pas surcharger la mémoire des élèves. Il a soin de multiplier les exemples, afin de rendre les principes plus saisissables; et, pour forcer l'élève à faire une juste et continuelle application des règles [...]. Le chapitre des verbes, point si essentiel et si difficile dans l'étude des langues étrangères, est peut-être celui qui est le mieux traité. Ce sujet est soumis à la méthode la plus claire, et les divisions en sont simples et naturelles. On y trouve tout ce qui est essentiel, tant pour ce qui regarde les verbes en général, que pour ce qui leur est particulier dans la langue espagnole $[\ldots]$. On doit penser que cette méthode si claire de l'auteur s'étend à la Syntaxe. Cette partie présente les règles qui n'ont pu trouver dans la première partie, parce qu'elles n'auraient servi qu'à embarrasser l'élève. Là l'auteur montre toutes les locutions particulières à la langue espagnole, toutes ses finesses, toutes ses délicatesses. Tout est simple quoique parfaitement raisonné. Chaque leçon est suivie d'exercices en français et en espagnol, afin de familiariser l'élève avec les difficultés de la langue qu'il veut apprendre (Lopes 1866: VII y VIII).

Por su parte, la Nouvelle méthode pratique de la langue espagnole es una selección de los contenidos gramaticales de la Nouvelle grammaire pratique et raisonnée de la langue espagnole expuestos en apenas ciento cincuenta páginas. La publicación de este manual, como se explica en el prefacio, se debe a la apuesta de los editores por "petites méthodes, refermant le mécanisme d'une langue, très faciles à suivre et à comprendre et permettant de se servir ensuite de grammaires plus complète»(Lopes 1873: II), pero junto al interés didáctico está también el económico, pues reconocen que «après le bon accueil dont le public a honoré la Nouvelle méthode pratique anglaise, nous ne pouvions pas nous dispenser de lui offrir une méthode analogue pour la langue espagnole» (1873: II).

En esta ocasión, Lopes, o su editor -que era ya Leroy-, estructuran la obra de la siguiente forma: 1) un capítulo sobre pronunciación; 2) un compendio sobre los «verbes et leur application»; 3) un resumen de algunas partes del discurso (formas y usos del artículo, del nombre sustantivo, del adjetivo, de los pronombres, de los adverbios, de la preposición y de la conjunción); 4) la nómina de los principales idiotismos del español; y 5) dos vocabularios alfabéticos con las palabras utilizadas en el método.

Este compendio, más breve que la gramática pero igual de claro y conciso, tenía un objetivo evidente: menos páginas, mismos contenidos; una suerte para los usuarios y, sobre todo, para el vendedor.

\section{Los cursos graduados: manuales de conversación, diálogos, lecturas y versiones}

Bajo el título Cours gradué Truchy publicó una serie de obras con diferentes contenidos destinadas a los niveles elementales de aprendizaje de lenguas. La primera, el Cours gradué de langue anglaise divisé en trois parties à l'usage des classes élémentaires (1829) que llegó a conocer más de treinta ediciones hasta comienzos del siglo $\mathrm{xx}$, incluía: $1^{\circ}$ choix de dialogues anglais et français sur toutes 
les circonstances particulières de la vie, $2^{\circ}$ recueil de locutions et d'expressions, dans lesquelles les étrangers sont exposés à faire des contre-sens, $3^{\circ}$ riche vocabulaire de tous les mots les plus usités, classés par matière, suivi de tables comparatives des poids, mesures et monnaies, etc, des deux pays. A l'usage des maisons d'éducation.

Con el paso del tiempo, y ante el éxito del manual, Truchy decide publicar la primera parte del Cours de forma independiente con el título Manuel classique de conversations françaises et anglaises, en une série de dialogues destinés à faciliter la pratique de la conversation familière, servant aussi d'interprète fidèle aux voyageurs visitant la France et l'Angleterre ${ }^{10}$, y adaptarlo a otras lenguas: Manuel de conversations françaises et italiennes contenant: 1. Vocabulaires des mots usuels avec leurs exercices particuliers pour en faciliter l'emploie immédiat. 2. Petites phrases familières et élémentaires. 3. Dialogues sur différens sujets. On y a joint La conjugaison des verbes auxiliaires avoir et être avec des substantifs et des adjectifs. Des exercices sur un grand nombre de verbes réguliers des trois conjugaisons. La liste des verbes irréguliers des trois conjugaisons avec leurs exercices por Sforzosi de Rome (1831) ${ }^{11}$, Manuel de phrases françaises et allemandes, contenant de nombreux vocabulaires des mots les plus usités suivis chacun de petites phrases élémentaires accompagné de dialogues familiers, à l'usage des classes élémentaires por Stahl (1835), Manuel de conversations françaises et espagnoles, contenant un tableau synoptique d'Ortologie espagnole; verbes réguliers et irréguliers, avec les diverses aceptions des verbes ser et estar, et des dialogues sur un grand nombre de sujets por José M. ${ }^{a}$ Moralejo (1835) ${ }^{12}$.

El Manuel está formado por varios contenidos que se repiten, aunque con pequeñas modificaciones, en todas las versiones:

- una nomenclatura, un repertorio léxico temático que tiene como objetivo recoger en diferentes ámbitos designativos el léxico básico o usual de la lengua (Dieu et la création, Le temps, Les fêtes, Pour s'habiller, Les parties d'une maison, Pour manger et boire, Parenté, Professions, Fleurs et fruits, Quadrupèdes, oiseaux, poissons, Vertus et vices), «des mots les plus usités» como consta en la portada, y su ejemplificación o uso en frases cortas para facilitar la adquisición del vocabulario. Así, por ejemplo, las partes del cuerpo se ejemplifican con enunciados como "La tête est faible», "Il a été frappé au front», "On lui a crevé les yeux», «J'ai fermé les paupières», «La prunelle de l'œil droit»o «Il s'est cassé le nez»;

10 En el «Avis de l'éditeur» de la séptima reimpresión de la segunda edición del Manuel classique de conversations françaises et anglaises (Sadler 1847: v) explica las razones de este cambio: «le débit de plus de trente mille exemplaires de cet excellent ouvrage destiné à l'usage des classes élèmentaires, nous ont fait sentir depuis longtemps le besoin d'en donner une espèce de complément, mais d'un degré plus élevé et qui pût convenir aussi bien aux gens du monde qu'au voyageur ou à l'élève studieux».

11 La versión italiana se reimprimió y reeditó en 1836, en 1837, en 1843, en 1854, en 1864, en 1866, en 1876 y en 1891.

12 La versión española volvió a aparecer, corregida y aumentada, en 1847 y en 1862. 
- nociones de gramática, sobre todo pronunciación y resúmenes del sistema verbal;

- diálogos (Pour saluer, La visite, Le déjeûner, Avec un libraire, Pour acheter différens objets, Surl'heure, Sur le temps, Sur les chemins de fer), que también resultaban muy útiles para el aprendizaje de lenguas por la explotación que podía hacerse de ellos en todos los niveles lingüísticos: fonético (vienen acompañados de la transcripción), morfológico (conjugaciones verbales, pronombres personales), sintáctico (la disposición en columnas paralelas de los diálogos posee una función contrastiva de la forma distinta de construir frases en cada una de las lenguas del compendio), semántico (con el léxico contextualizado o empleado en una situación determinada) y pragmático (es requisito en estas guías incorporar factores extralingüísticos determinantes en el uso del lenguaje como la situación, el conocimiento del mundo o el contexto socio-cultural) ${ }^{13}$ (Sáez 2005);

- y, en algunas ediciones, Phrases familières y nomenclaturas sobre el comercio o la marina.

Una vez más, Truchy aprovecha materiales viejos para su método: las nomenclaturas, las descripciones gramaticales o los diálogos se venían utilizado en la enseñanza de lenguas desde la Edad Media (Alvar Ezquerra 2013, Chevalier 1988, Colombo 1992 y 1998, Sánchez Pérez 1992); Truchy solo se limita a adaptarlos a las necesidades de sus lectores.

Pero los Cours gradués no solo contenían manuales de frases, «vocabulaires des mots les plus usités» y diálogos, sino que con este título se publicaron también lecturas y versiones para completar, sobre todo en las aulas de las clases elementales, la enseñanza gramatical y léxica. Es así como ven la luz el Cours gradué de langue anglaise. Cours élémentaire. Deuxième partie ou Petit cours de versions. Suivi d'un dictionnaire anglais-français (1835, Percy Sadler), el Cours gradué de langue anglaise. Cours élémentaire. Troisième partie ou Petit cours de thèmes. Suivi d'un dictionnaire français-anglais de tous les mots qui s'y trouvent (1835, Percy Sadler) ${ }^{14}$, el Cours gradué de langue allemande ou Recueil de petites versions allemandes à l'usage des classes élémentaires, contenant un

13 La utilidad de los diálogos en la enseñanza de lenguas determinó que Truchy publicara otros de forma independiente: en 1836 aparece la Guide de la conversation française et anglaise, à l'usage des voyageurs et des étudiants (Léon Smith), en 1837 los Dialogues familiers français-italiens, à l'usage des étudiants et de ceux qui voyagent en Italie (A.Ronna), en 1838 los Dialogues familiers français-anglais, précédés d'un vocabulaire (Asborne de Chastelain), en 1839 los Dialogues familiers françaisallemands (J.C. Kriegk), en 1841 las Scènes du jeune âge (en anglais et en français), ou Conversations et entretiens familiers de jeunes enfants (Asborne de Chastelain), en 1851 Le Guide usuel de la conversation familière française et anglaise (S. Dixon) y en 1852 la Guide usuel de la conversation familière française et allemande (C. Wolters).

14 La primera parte es el Cours gradué de langue anglaise. Première partie: Manuel de phrases françaises et anglaises et anglaises contenant de nombreux vocabulaires des mots les plus usités, suivis chacun de petites phrases élémentaires servant d'exercices, précédés d'une série de leçons préparatoires (1829), de Percy Sadler. 
choix d'anecdotes et de morceaux historiques avec des notes françaises explicatives et suivi d'un dictionnaire allemand-français (1840, Weber), el Petit cours gradué de thèmes allemands (1844, André Wahl) o el Cours gradué de langue espagnole: lectura, versions, littérature et dictionnaire raisonné du texte dans l'ordre des matières (1845, Pablo Valdemoros y Álvarez).

En este último caso, Pablo Valdemoros explica que la riqueza y la variedad de la Literatura española le han animado a recopilar «les passages qui m'ont paru les plus remarquables, soit par le style, soit par la pensée» (1845: VIII). La primera parte contiene treinta y seis lecciones, correspondientes a las treinta y seis semanas que tiene el curso escolar, que recogen textos sobre la historia de España, sobre el Quijote de Cervantes, sobre Motezuma, sobre la entereza del rey Enrique VIII, reflexiones sobre el matrimonio, exordio del marqués de Santa Cruz, la ribera del Tajo, Santa Teresa de Jesús a Diego Hurtado de Mendoza, aventura del jarrón de vino, discurso del moro Aben-Jauhar o sobre la grandeza y decadencia de España; la segunda parte, en donde «l'action du professeur devait être plus libre» (Valdemoros 1845: IX), está integrada por varios textos en prosa (heroicidad de Guzmán el Bueno en Tarifa, la novia soberbia domada, Rinconete y Cortadillo, la corrida de toros) y en verso (Salicio y Nemoroso, el burro flautista, el juez escrupuloso, la mujer de Caupolicano, el camello y la pulga), que permiten al alumno conocer diferentes géneros, estilos y obras escritos en lengua española. Para facilitar la lectura y comprensión de los textos, Valdemoros incluye en el primero una traducción interlineada y, en las últimas páginas, un diccionario con «les locutions, les idiotismes, les mots même dont la signification littérale diffère de celle du texte» (Valdemoros 1845: X) y su correspondiente explicación.

La utilización de fragmentos literarios, de autoridades, en la enseñanza de lenguas tampoco es original en la historia: desde la antigüedad clásica se utilizaban como modelos de buen uso, y podían ser explotados en la clase de lengua extranjera con diversas finalidades (lectura, traducción, léxico, gramática, cultura e historia, variantes lingüísticas... $)^{15}$, lo que, sin duda alguna, contribuía a la adquisición de la nueva lengua

j'ai voulu seulement distraire mes lecteurs et leur donner la conscience de leur force, en leur prouvant ainsi que je leur ai fait prendre, non seulement le chemin

15 Después de la introducción, y en letra más pequeña, Valdemoros da unos consejos sobre la utilidad y el provecho de este manual: «j'engage les professeurs, après faire lire et traduire la leçon deux fois, si le temps le permet, à répéter eux-mêmes à haute voix (les libres des élèves étant fermés) les phrases contenant des idiotismes, des locutions ou des tournures particulaires au génie de la langue espagnole, et à les faire traduire en français d'après leur seule audition. Quand les élèves seront suffisamment avancés, le professeur pourra ajouter à cet exercice la contra-partie, c'est à dire, faire traduire en espagnol les phrases de la version qu'il dira en français. Outre que ces exercices sont un contrôle certain du travail des élèves, ils les disposent efficacement et sans transition à la langue parlée, qui est, selon moi, le but principal qu'on doit se proposer dans l'étude d'une langue vivante» (Valdemoros 1845: XII). 
le plus cort et le plus sûr, mais encore le plus aplani, puisqu'ils seront arrivés au terme du voyage presque sans s'en douter. (Valdemoros 1845: XI)

\section{Guías de conversación}

En 1843 aparece L'anglais tel qu'on le parle ou recueil de conversations anglaises et françaises avec la prononciation anglaise figurée par des sons français à l'usage des français qui vont à Londres, publicación que queda justificada en el prefacio (Cumberworth 1843: V-VI):

Quand on considère le nombre et la variété de Manuels et de Guides de la conversation française et anglaise, on ne peut guère attendre d'une nouvelle publication que l'avantage d'un bon choix et un arrangement tout à fait convenable au sujet.

Dans l'ouvrage que nous offrons au public, nous nous sommes particulièrement attaché à éviter les répétitions inutiles, en les remplaçant par ce qu'il peut y avoir de plus important à connaître pour un étranger, et en lui donnant la formule la plus simple pour bien exprimer ce qu'il désire faire comprendre.

Volumen encuadernado en octavo de apenas ciento setenta páginas que reúne unas observaciones sobre pronunciación inglesa, unos «dialogues françaisanglais, avec la prononciation anglaise figurée», unos "vocabulaires usuels», también con la pronunciación figurada, y unas tablas con las correspondencias de las monedas, pesos y medidas en ambas lenguas, esto es, «nous avons eu soin d'abord de choisir les sujets presentant le plus d'utilité aux voyageurs qui visitent l'Angleterre» (Cumberworth 1843: VI).

Con el paso del tiempo, Truchy primero y los hermanos Leroy después ampliaron el contenido de esta guía de conversación, la volvieron a imprimir (llegó a tener quince ediciones: 1843, 1847, 1853, 1873, 1880, 1883, 1889, 1892, $1903 . .$.$) y la tradujeron a varias lenguas.$

\begin{tabular}{|l|l|}
\hline \multicolumn{1}{|c|}{ Título, año, autor } & \multicolumn{1}{|c|}{ Finalidad } \\
\hline $\begin{array}{l}\text { L'allemand tel qu'on le parle ou recueil de } \\
\text { conversations allemandes et françaises avec } \\
\text { la prononciation allemande figurée par des } \\
\text { sons français à l'usage des français qui vont en } \\
\text { Allemagne, 1845, A. Wahl'16 }\end{array}$ & $\begin{array}{l}\text { «Le but de ce petit ouvrage est d'expliquer la } \\
\text { manière de prononcer les lettres, les syllabes } \\
\text { et les mots allemands, et de faciliter l'étude } \\
\text { d'une langue si nécessaire au commerce et } \\
\text { aux sciences» }(\mathrm{p} . \mathrm{V})\end{array}$ \\
\hline $\begin{array}{l}\text { L'espagnol tel qu'on le parle ou recueil de } \\
\text { conversations espagnoles et françaises avec } \\
\text { la prononciation espagnole figurée par des } \\
\text { sons français à l'usage des français qui vont en } \\
\text { Espagne, 1867, J. M. Lopes }\end{array}$ & $\begin{array}{l}\text { «Nous avons voulu venir en aide à ceux qui } \\
\text { ayant des notions imparfaites de la grammaire } \\
\text { espagnole, ou les ayant acquises sans le } \\
\text { secoun maître, ignorent complétement } \\
\text { le mécanisme de la pronociation castillane» } \\
\text { (p. V) }\end{array}$ \\
\hline
\end{tabular}

16 La guía de conversación para el alemán fuen reimpresa, hasta el siglo xx, en 1845, $1858,1872,1875,1879,1882,1889,1893,1898$ у 1900. 


\begin{tabular}{|c|c|}
\hline $\begin{array}{l}\text { L'italien tel qu'on le parle ou recueil de } \\
\text { conversations italiennes et françaises avec la } \\
\text { prononciation italienne figurée par des sons } \\
\text { français à l'usage des français qui vont en Italie, } \\
1868, \text { C. I. Rapelli17 }\end{array}$ & $\begin{array}{l}\text { «Nous ne complétions pas le cadre des } \\
\text { quatre langues modernes les plus usuelles, } \\
\text { en offrant au public un ouvrage analogue sur } \\
\text { l'italien» (p. V) }\end{array}$ \\
\hline $\begin{array}{l}\text { Le portugais tel qu'on le parle ou recueil de } \\
\text { conversations portugaises et françaises avec } \\
\text { la prononciation portugaise figurée par des } \\
\text { sons français à l'usage des français qui vont en } \\
\text { Portugal, 1891, Soares da Silva }\end{array}$ & $\begin{array}{l}\text { "Nous avons voulu venır en alde a ceux qul, } \\
\text { portugaise, ou les ayant acquises sans le } \\
\text { secours d'un maître, ignorent complétement } \\
\text { le mécanisme de la pronociation portugaise. } \\
\text { Voilà le but de Le portugais tel qu'on le parle. Ce } \\
\text { but est justifié par les services qu'ont rendus } \\
\text { aux voyageurs cinq éditions de l'Anglais, de } \\
\text { l'Allemand, de l'Italien, de l'Espagnol tel qu'on } \\
\text { le parle» (p. V) }\end{array}$ \\
\hline $\begin{array}{l}\text { Le russe tel qu'on le parle ou recueil de } \\
\text { conversations russes et françaises avec } \\
\text { la prononciation russe figurée par des sons } \\
\text { français à l'usage des français qui vont en } \\
\text { Russie, 1892, Wladimir Stavenhagen }\end{array}$ & $\begin{array}{l}\text { «Le but de ce petit ouvrage est d'expliquer la } \\
\text { manière de prononcer les lettres, les syllabes } \\
\text { et les mots russes, et de faciliter l'étude d'une } \\
\text { langue si nécessaire au commerce et aux } \\
\text { sciences» (p. V). }\end{array}$ \\
\hline
\end{tabular}

Pese a que el contenido de las guías de conversación de la casa Truchy cambió desde su primera aparición, la primera edición de L'anglais tel qu'on le parle recoge la esencia de estas guías de conversación, a saber:

a) Un breve tratado sobre pronunciación repartido en tres secciones: la tabla de los sonidos vocálicos (donde se proponen trascripciones como « $a$ fermé dans fate prononcez féte», "a ouvert bref dans fat prononcez făte», «a ouvert long dans far prononcez fàr», «a grave bref dans was prononcez ouăze», « $a$ grave long dans wall prononcez ouāll»), la tabla de los sonidos consonánticos («b dans bag prononcez bag'», "d dans dog prononcez dŏg», " $p$ dans put prononcez poute», «th (aigu ou dur) dans three prononcez thrî», "th (grave ou doux) dans them prononcez themm») y unas observaciones sobre el sistema adoptado para «figurer la prononciation» («Dans la prononciation figurée, nous indiquons l’ă et l'ŏ graves brefs, et l'ě gutural ou muet par une petite ligne courbe, et l'ā et l'ō graves longs par une petite ligne droite», «NG. Cette double consonne a un son nasal très sonore, qui se rapproche de celui de l'n français, après les voyelles $a$, $o$, excepté qu'il faut presser le milieu de la langue contre le palais, comme pour le $g$ dur, et laisser passer le son principalement par le nez, exemple: sang, hang, sing, bring. Observez que les voyelles $a$ et $i$, que précèdent le $n g$, ont les sons purs de l'alphabet français») ${ }^{18}$, dado que, como se explica en el prefacio, resulta indispensable

17 La guía de conversación del italiano volvió a reimprimirse en 1874, 1879, 1883, 1887, 1891,1900 y 1902.

18 En ocasiones, las observaciones iniciales sobre pronunciación resultan claramente insuficientes, y es necesario incluir, en notas a pie de página, otras indicaciones sobre la pronunciación figurada; así, por ejemplo, en la página 1 se dice «Nous faisons remarquet ici que tous les $e$ finals qui sont dans la prononciation figurée doivent être 
ajouter la prononciation figurée de tous les mots anglais en regard du texte; car si l'on ne prononçait pas les mots et les syllabes avec l'accent convenible, il arriverait que souvent on ne serait pas compris, quels que fussent la connaissance et l'usage que l'on eût de la langue anglaise. Mais ce qui était plus essentiel encore, c'était de la bien figurer, pour éviter les inconvénients qui résulteraient du contraire. Prenons, par exemple, les verbes to sleep, dormir, to weep, pleurer, qui doivent être figurés tou slîpe, tou ouîpe; tandis que si on les figurait tou slip, tou ouip, ils signifieraient glisser, fouetter (Cumberworth 1843: VII)

Con el «secours d'un très-petit nombre de leçons d'un maître» y con las indicaciones fonéticas incluidas en estas primeras páginas, cualquier persona podrá, según Truchy, pronunciar y leer esta guía «aussi bien que puisse l’espérer un étranger» (Cumberworth 1843: 8).

b) Varios diálogos que, tal y como se afirma en el prefacio de las siguientes ediciones, están dispuestos «de manière qu'on puisse les appliquer à presque toutes les circonstances dans lesquelles un voyageur peut se trouver dans le cours de son voyage ou de son séjour dans une ville». En la edición de 1843 de L'anglais tel qu'on le parle hay veintidós diálogos que tratan sobre «Avec le capitaine du bateau à vapeur», "Sur le bateau à vapeur», "Arrivée à Londres», "Arrivée à l'hôtel», "Le soir, le coucher», "Le matin, le lever», "Le déjeuner», "Sur les édifices publics à Londres», "Visite, invitation à dîner», "Le dîner», «Le dessert», «Le thé», "Au spectacle», "Chez un libraire», "Chez le coutelier», "Chez un horloger», "Chez un marchand de nouveautés», «Dans un restaurant», «Au Bureau des diligences», "Le départ», «Le voyage de Londres à Douvres» $\mathrm{y}$ «L'arrivée à l'auberge de Douvres» ${ }^{19}$.

prononcés comme $e$ muet dans les mots français fiole, soupe, mille, en ne le faisant pas plus entendre que dans les mots $v o l$, bol, etc.», y en la página 11 «rappelez-vous toujours que les $n$ ' avec apostrophe doivent toujours être prononcés comme doubles, pour éviter le son nasal».

19 Copio a continuación uno de los diálogos para mostrar la posibles explotaciones didácticas de estos textos:

Quels sont les édifices les plus
intéressants à voir?
Vous pourrez d'abord aller voir la
Tour, I'hôtel de la Monnaie et la
douane, qui sont du côté de l'est
de Londres, et tous dans le même
quartier.
Y a-t-il quelque chose de curieux
dans cette partie de Londres qu'on
appelle la Cité?
Les principaux édifices publics
sont:
L'Hotel de la Compagnie des Indes
orientales, la banque, la bourse,
I'hôtel du lord-maire, I'hôtel de
ville, la grande poste et l'église de
Saint-Paul.

8.- Sur les édifices publics à Londres

What are the buildings most worth seeing?

You may first go and see the

Tower, the Mint and the cus-

tom-house, which are on the

eastern side of London, and all in

the same neighbourhood.

Is there any thing curious in that

part of London called the city?

The principal edifices are:

The East-India-House, the Bank, the Royal-Exchange, the Mansion-House, Guildhall, the Post-Office and St-Paul's-Church.
Houăte are thi bild-igne-ze môst ouorth si-igne?

Yoù mé forste gè an'd sì thi Tăou-ěr thi Min't, an'd thi costom-hăouce, houitche àre ŏne thi ìst-ěrn saïde ŏv'Lon'-don', and âll ine thi séme né-bor-houde. Ize thére en'-ni THingne kiour i-ŏce in that pàrt ŏv' Lon'-don' kāll'd thi cit-i?

Thi princ'-cip-al ed-i-fiss-eze àre: Thi îste-In'd-ia-hăouce, thi Bangke, thi rŏî-al-Extchen'dje, thi Man's-chon-hăouce, Guild-hāll, thi Pòst-ŏf-fice, an'd sén'te Pāl'ze tchortche. 
c) Una nomenclatura o «Vocabulaires usuels» que dedica epígrafes a «Plats qu'on trouve généralement dans les restaurants anglais ${ }^{20}$ », "Les jours de la semaine», «Les mois de l'année, saisons, éléments», «Le temps», «Des meubles», «De la table», «Des habits», «Habillements de femmes», «La parenté», «Jeux et divertissements», «De la chasse aux bêtes et aux oiseaux, etc.», "Jeux de hazard», «Métiers et occupations», «Nombres cardinaux», «Nombres ordinaux» $\mathrm{y}$ «Tables pour les monnaies, les poids et mesures».

Se trata, en definitiva, de recoger el léxico básico del inglés para ofrecérselo a los franceses que tuviesen interés en viajar a Inglaterra, y hacerlo a través de una ordenación lógica que ponía juntas voces cercanas por lo designado, facilitaba la adquisición.

\section{Conclusiones}

Las obras descritas en las páginas anteriores muestran la labor realiazada por la editorial de J.H. Truchy, quien se propuso confeccionar un método de enseñanza de lenguas modernas que resultase práctico y útil a todos aquellos franceses que quisieran cruzar las fronteras de Inglaterra, Alemania, Portugal, España, Rusia o Italia, así como aquellos que deseasen aprender otra lengua dentro de las aulas. Las sucesivas versiones, ediciones y reimpresiones de la mayoría de sus obras confirman, por un lado, el provecho que obtuvieron editores y libreros de la demanda que suponían los cambios producidos en el nuevo escenario económico europeo, $y$, por otro, la importancia en esta centuria de la industria del libro en la enseñanza de segundas lenguas, realidad que no puede ni debe permanecer al margen de la lingüística.

Aunque la mayoría de estos materiales no incluyen contenidos nuevos ni originales (ni las gramáticas, ni los manales de correspondencia, ni los diálogos, ni los vocabularios, ni las nomenclaturas), constituyen un capítulo interesante en la historia de la enseñanza de lenguas del siglo xIX, y permiten valorar la labor de editores e impresores en la creación y elaboración de materiales didácticos.

\section{Bibliografía}

\section{Fuentes primarias}

Cumberworth T., 1843, Langlais tel qu'on le parle ou recueil de conversations anglaises et françaises avec la prononciation anglaise figurée par des sons français à l'usage des français qui vont à Londres, Paris, Truchy.

20 Es frecuente encontrar en el capítulo dedicado a la gastronomía de cada país notas con los ingredientes de un plato o información sobre el plato equivalente en Francia; en el caso del inglés, por ejemplo, se hace constar que «le rump-steak est un beef-steak de la première qualité» o que «l'alamode-beef ressemble peu au mets qu'on appelle bouf à la mode en France, c'est une espèce de consommé que l'on sert avec de la viande dedans». 
Lopes J.M., (1835 [1847, 1862]), Manual de conversaciones francesas y españolas, Paris, Truchy.

Lopes J.M., 1864, Nueva correspondencia comercial francesa-español, que contiene la definición y explicación del lenguage comercial y económico, Paris, Truchy.

Lopes J. M., 1866, Nouvelle grammaire pratique et raisonnée de la langue espagnole, Paris, Truchy.

Lopes J. M., 1867, L'espagnol tel qu'on le parle ou recueil de conversations espagnoles et françaises avec la prononciation espagnole figurée par des sons français à l'usage des français qui vont en Espagne, Paris, Truchy.

Lopes J.M., 1873, Nouvelle méthode pratique de la langue espagnole, Paris, Truchy. Lopes J.M., 1889, El francés tal como se habla o colección de conversaciones francesas y españolas con la pronunciación francesa figurada con sonidos españoles para uso de los españoles y americanos que vienen a Francia, Paris, hermanos Leroy, sucesores de Truchy.

Rapelli C. I., 1868, L'italien tel qu'on le parle ou recueil de conversations italiennes et françaises avec la prononciation italienne figurée par des sons français à l'usage des français qui vont en Italie, Paris, Truchy.

Sadler P., 1847, Manuel classique de phrases françaises et anglaises, Paris, Truchy.

Soares da Silva, 1891, Le portugais tel quion le parle ou recueil de conversations portugaises et françaises avec la prononciation portugaise figurée par des sons français à l'usage des français qui vont en Portugal, Paris, hermanos Leroy, sucesores de Truchy.

Stavenhagen W., 1892, Le russe tel qu'on le parle ou recueil de conversations russes et françaises avec la prononciation russe figurée par des sons français à l'usage des français qui vont en Russie, Paris, hermanos Leroy, sucesores de Truchy.

Valdemoros y Álvarez P., 1845, Cours gradué de langue espagnole: lectura, versions, littérature et dictionnaire raisonné du texte dans l'ordre des matières, Paris, Truchy.

Wahl A., 1845, Lallemand tel quion le parle ou recueil de conversations allemandes et françaises avec la prononciation allemande figurée par des sons français à l'usage des français qui vont en Allemagne, Paris, Truchy.

Fuentes secundarias

Alvar Ezquerra M., 2013, Las nomenclaturas del español. Siglos XV-XIX, Madrid, Liceus.

Bruña Cuevas M., 2008, Philologia Hispalensis. Lexicografía bilingüe y plurilingüe del español (siglos XV-XIX), Sevilla, Universidad de Sevilla [especialmente los trabajos de J. J. Martínez Egido, «Origen y desarrollo positivo de la lexicografía español-italiano (siglos XVI-XIX)», D. Messner, "Los diccionarios bilingües español-portugués desde el principio hasta el siglo XIX», M. J. Corvo Sánchez, «Breve historia de la lexicografía bilingüe española y alemana hasta el siglo XIX» y M. Bruña Cuevas, «La producción lexicográfica con el español y el francés durante los siglos XVI a XIX»]. 
Calero Vaquera M. ${ }^{a}$ L., 1986, Historia de la gramática española (1847-1920). De A. Bello a R. Lenz, Madrid, Gredos.

Cazorla Vivas C., 2002, Lexicografía bilingüe con el español y el francés de los siglos XVIII y XIX, Madrid, Universidad Complutense de Madrid.

Chevalier J.-Cl., 1988, «Les dialogues médiévaux. Origines, filiations, méthodes. Quelques pistes», Documents pour l'enseignement du français langue étrangère ou seconde, 22, p. 17-26.

Choppin A., 1992, Les Manuels scolaires: histoire et actualité, Paris, Hachette.

Choppin A. (dir.), 1995, Les Manuels scolaires en France de 1789 à nos jours: les Manuels d'espagnol, Paris, INRP.

Colombo Timelli M., 1992, «Dictionnaires pour voyageurs, dictionnaires pour marchands ou la polyglossie au quotidien aux XVI ${ }^{\mathrm{e}}$ et $\mathrm{XVII}^{\mathrm{e}}$ siècles», Linguisticae Investigationes, XVI, p. 395-420.

Colombo Timelli M., 1998, «Dialogues et phraséologie dans quelques dictionnaires plurilingues du $\mathrm{XVI}^{\mathrm{e}}$ siècle», Documents pour l'enseignement du français langue étrangère ou seconde, 22, p. 27-59.

Cooper-Richet D., 1999, «La librairie étrangère à Paris au XIX ${ }^{e}$ siècle», in: Actes de la recherche en sciences sociales, 126/1, p. 60-69.

Cooper-Richet D., 2002, "L'imprimé en langues étrangères à Paris au XIX siècle: lecteurs, éditeurs, supports», Revue française d'histoire du libre, 116/2, p. 203-235.

Fernández Fraile M.E. y Suso López J., 1999, La enseñanza del francés en España (1767-1936). Estudio histórico: objetivos, contenidos, procedimientos, Granada, Método Ediciones.

García Aranda M. ${ }^{a}$ Á. 2012a, «Los materiales empleados en la enseñanza gramatical del español en Francia: las Gramáticas y Métodos de José M. Lopes (1866, 1873 y 1892)», Revista Argentina de Historiografía Lingüistica, IV/1, p. 1-21.

García Aranda M.a Á. 2012b, «El español de los negocios en el siglo xix: la Nueva correspondencia comercial francesa-española de José M. Lopes (Paris, Truchy, 1864)», Bulletin Hispanique, 114/2, p. 853-872.

García Aranda M. ${ }^{a}$ Á. 2014, «Un nuevo método de estudio de lenguas extranjeras en el siglo xix: las guías prácticas de conversación y pronunciación», Anuario de Estudios Filológicos, 37, p. 41-69.

Institut français de l'education, http://www.inrp.fr/emma/web/index.php.

Quemada B., 1967, Les Dictionnnaires du français moderne, 1539-1863, Paris/ Bruselles/Montreal, Didier.

Sáez Rivera D., 2005, «La explotación pedagógica del diálogo escolar en la didáctica del español (siglos XVI-XIX)», in: M.A. Castillo Carballo (coord.), Las gramáticas y los diccionarios en la enseñanza del español como segunda lengua, deseo y realidad. Actas del XV Congreso Internacional de ASELE. Sevilla 22-25 de septiembre de 2004, Sevilla, Universidad de Sevilla, p. 792-798.

Sánchez Pérez A., 1992, Historia de la enseñanza del español como lengua extranjera, Madrid, SGEL. 